\title{
Corrigendum
}

\section{Flavanone-rich citrus beverages counteract the transient decline in postprandial endothelial function in humans: a randomised, controlled, double-masked, cross-over intervention study - CORRIGENDUM}

\author{
Authors \\ Catarina Rendeiro ${ }^{1} \dagger$, Honglin Dong ${ }^{1} \ddagger$, Caroline Saunders ${ }^{2}$, Laura Harkness ${ }^{3}$, Melvin Blaze ${ }^{4}$, Yanpeng Hou ${ }^{4}$, Ronald L. Belanger ${ }^{3}$, \\ Giulia Corona $^{1} \S$, Julie A. Lovegrove ${ }^{1}$ and Jeremy P. E. Spencer ${ }^{1 *}$ \\ ${ }^{1}$ Department of Food and Nutritional Sciences, School of Chemistry, Food and Pharmacy, University of Reading, PO Box 226 , Reading \\ RG2 6AP, UK \\ ${ }^{2}$ PepsiCo R+D Nutrition, PepsiCo Inc., Reading RG2 6UW, UK \\ ${ }^{3}$ Global R+D Nutrition, PepsiCo Inc., Valhalla, NY 10595, USA \\ ${ }^{4}$ PepsiCo R+D Biological \& Discovery Analytics, PepsiCo Inc., New Haven, CT 06511, USA
}

*Corresponding author: J. P. E. Spencer, email j.p.e.spencer@reading.ac.uk

$\dagger$ Present address: Beckman Institute for Advanced Technology, University of Illinois UC, 405 Mathews Av, Urbana, IL 61802, USA.

$\ddagger$ Present address: School of Life Sciences, Faculty of Health and Life Sciences, Coventry University, Coventry, UK.

§resent address: Life Sciences Department, Whitelands College, University of Roehampton, Holybourne Avenue, London, UK.

Epub 2017 Jan 9

DOI: $10.1017 /$ S0007114516004219

Original text and correction

\section{The Author list above should be corrected to the following:}

Catarina Rendeiro ${ }^{1} \dagger$, Honglin Dong ${ }^{1} \ddagger$, Caroline Saunders ${ }^{2}$, Laura Harkness ${ }^{3}$, Melvin Blaze ${ }^{4}$, Yanpeng Hou ${ }^{4}$, Ronald L. Belanger ${ }^{3}$, Vincenzo Altieri ${ }^{4}$, Michael A. Nunez ${ }^{4}$, Kim G. Jackson ${ }^{1}$, Giulia Corona ${ }^{1} \S$, Julie A. Lovegrove ${ }^{1}$ and Jeremy P. E. Spencer ${ }^{1 *}$

${ }^{1}$ Department of Food and Nutritional Sciences, School of Chemistry, Food and Pharmacy, University of Reading, PO Box 226, Reading RG2 6AP, UK

${ }^{2}$ PepsiCo R+D Nutrition, PepsiCo Inc., Reading RG2 6UW, UK

${ }^{3}$ Global R+D Nutrition, PepsiCo Inc., Valhalla, NY 10595, USA

${ }^{4}$ PepsiCo R+D Biological \& Discovery Analytics, PepsiCo Inc., New Haven, CT 06511, USA

"Corresponding author: J. P. E. Spencer, email j.p.e.spencer@reading.ac.uk

$†$ Present address: Beckman Institute for Advanced Technology, University of Illinois UC, 405 Mathews Av, Urbana, IL 61802, USA. $\ddagger$ Present address: School of Life Sciences, Faculty of Health and Life Sciences, Coventry University, Coventry, UK.

§Present address: Life Sciences Department, Whitelands College, University of Roehampton, Holybourne Avenue, London, UK.

\section{Author Contributions:}

C. R.: coordinated and conducted the study, undertook all FMD measurements, performed data analysis and wrote the manuscript; H. D.: coordinated and conducted the study; C. S.: designed and coordinated the study and wrote the manuscript; L. H.: designed the study; R. L. B.: conducted study drinks analysis; M. B., Y. H., V. A., M. A. N.: conducted plasma flavanone analysis; G. C.: conducted NO species measurements and analysis; J. A. L. and K. G. J.: co-investigated the study and was involved in experimental design; J. P. E. S.: principal investigator, involved in the experimental design and writing of the manuscript. All authors reviewed the manuscript. C. S. works as a Senior Scientist at PepsiCo Inc., L. H. works as a Senior Director at Global R\&D Nutrition at PepsiCo Inc., R. L. B., M. B., Y. H. work as Principle Scientists at PepsiCo Inc., V. A., M. A. N., works as a Scientist at PepsiCo Inc., The other authors declare no conflicts of interest. 